\title{
Agents Deliberating over Action Proposals Using the ProCLAIM Model
}

\author{
Pancho Tolchinsky ${ }^{1}$, Katie Atkinson ${ }^{2}$, Peter McBurney ${ }^{2}$, \\ Sanjay Modgil ${ }^{3}$, and Ulises Cortés ${ }^{1}$ \\ ${ }^{1}$ Knowledge Engineering \& Machine Learning Group, Technical University of Catalonia, Spain \\ ${ }^{2}$ Department of Computer Science, University of Liverpool, Liverpool, UK \\ ${ }^{3}$ Advanced Computation Lab, Cancer Research UK
}

\begin{abstract}
In this paper we propose a dialogue game for agents to deliberate over a proposed action. The agents' dialogue moves are defined by a structured set of argument schemes and critical questions (CQs). Thus, a dialogue move is an instantiated scheme (i.e. an argument) or a CQ (i.e. a challenge on the argument instantiated in the scheme). The proposed dialogue game formalises the protocol based exchange of arguments defined in the ProCLAIM model. This model provides a setting for agents to deliberate over whether, given the arguments for and against, a proposed action is justified or not.
\end{abstract}

\section{Introduction}

Many domains illustrate requirements for multi-agent deliberation over a course of action (e.g., the medical domain [12]). These requirements include the distributed nature of the data relevant to the decision making, the specialist nature of agents involved in the deliberative process, their geographical distribution and the need to maintain independence between these entities. However, the inevitable presence of uncertainty and disagreement amongst specialist agents suggests that any model of distributed reasoning should account for conflicts that arise during deliberation. Logical frameworks for argumentation based dialogue have in recent years emerged as one of the most promising paradigms for formalising reasoning of the above kind (e.g. [10]2]). Such frameworks provide a principled way in which to structure the exchange of, reasoning about, and evaluation of the arguments for proposals, between human and or automated agents. Exchanged arguments claiming beliefs and or proposals for action are evaluated on the basis of their conflict based interactions, and their relative strengths, in order to determine a preferred course of action. One of the most promising approaches is based on an argument schemes and critical questions approach (S \& CQ) to argumentation over action [3]. Argument schemes, as described in the informal logic literature (e.g. [16]), are used to classify different types of argument that embody stereotypical patterns of reasoning. Instantiations of argument schemes can be seen as providing a justification in favour of the conclusion of the argument. The instantiated scheme (what we term an 'argument') can be questioned (attacked) through posing critical questions associated with the scheme. Each critical question can itself be posed as an attacking argument instantiating a particular scheme. This scheme is then itself subject to critical questioning. Computational accounts of the S \& CQ approach to argumentation over action [4]

H.-D. Burkhard et al. (Eds.): CEEMAS 2007, LNAI 4696, pp. 32 41, 2007.

(C) Springer-Verlag Berlin Heidelberg 2007 
have a number of advantages. The S \& CQ effectively map out the relevant space of argumentation, in the sense that for any argument they identify the valid attacking arguments from amongst those that are logically possible. They also provide a natural basis for structuring argumentation based dialogue protocols. Indeed, protocols based on the S \& CQ approach to argumentation over action [3] have been defined [5]. The latter work provides an abstract framework that can be instantiated and specialised for use in real world domains.

The primary contribution of this paper is that it proposes such a specialisation. A dialogue game for agents to argue over the appropriateness of an action is proposed for use within the ProCLAIM model (although the game is not inherently restricted to such use). This model posits the use of other components and knowledge sources required for practical and useful realisation of such a specialisation. In the following section we introduce the ProCLAIM model. In $\S 3$ we briefly describe one of the model's application scenarios, viz. human organ transplantation. In $\$ 4$ we define the dialogue game. In $\$ 5$ we present our intended future work, and in 6 we give our conclusions.

\section{The ProCLAIM Model}

The ProCLAIM model's primary component is a mediator agent $(M A)$. Three tasks are defined for the $M A: 1)$ The $M A$ guides proponent agents as to what are their legal dialectical moves at each stage in a dialogue; 2) the $M A$ also decides whether submitted arguments are valid (in the sense that instantiations of schemes are relevant w.r.t. the domain of discourse); 3) the $M A$ evaluates the submitted valid arguments in order to provide an assessment of the appropriateness of the proposed action. In order to undertake these tasks, $M A$ makes use of four knowledge resources, as shown diagrammatically in fig. (1) and also described below:

Argument Scheme Repository (ASR): In order to direct the proponent agents in the submission and exchange of arguments, the $M A$ references a repository of argument schemes and their associated CQs. The schemes and CQs are instantiated by agents to construct arguments, and effectively encode the full 'space of argumentation', i.e., all possible lines of reasoning that should be pursued w.r.t a given issue.

Guideline Knowledge (GK): This component enables the $M A$ to check whether the arguments comply with the established knowledge, by checking what the valid instantiations of the schemes in ASR are (the ASR can thus be regarded as an abstraction of the GK). This is of particular importance in safety critical domains where one is under extra obligation to ensure that spurious instantiations of argument schemes should not bear on the outcome of any deliberation.

Case-Based Reasoning Engine (CBRe): This component enables the $M A$ to assign strengths to the submitted arguments on the basis of their associated evidence gathered from past deliberations, as well as provide additional arguments deemed relevant in previous similar situations (see [15]).

Argument Source Manager (ASM): Depending on the source from whom the arguments are submitted, the strengths of these arguments may be readjusted by the $M A$. Thus, this component manages the knowledge related to, for example, the agents' roles and/or reputations. 


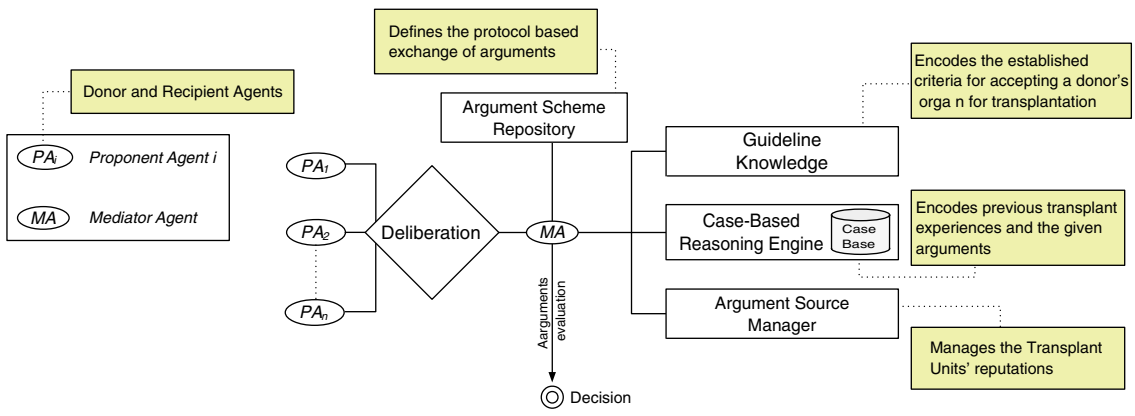

Fig. 1. ProCLAIM's Architecture. Shaded boxes identify the model's constituent parts specialised for the transplant scenario introduced in $\overline{3}$

The deliberation begins with one of the agents submitting an argument proposing an action. The $M A$ will then guide the proponent agents in the submission of further arguments that will attack or defend the justification given for the proposed action. Each submitted argument instantiates a scheme of the ASR. The $M A$ references the ASR in order to direct the proponent agents as to what their legal argument moves are at each stage of the deliberation. The strength 11 of each submitted argument is determined by the $M A$ in referencing the other three knowledge resources, viz. GK, CBRe and ASM. Given all the accepted arguments, their strength and their interactions (based on the attack relation, see fig. $4 \mathrm{~b}$ ) the $M A$ then applies Dung's seminal calculus of opposition [8] to determine the justified or winning arguments. Hence, if the initial argument is a winning argument, the proposed action is deemed appropriate, otherwise, it is rejected.

We now introduce one of our working scenarios, organ transplantation, in order to illustrate the use of ProCLAIM, and in particular the dialogue game.

\section{The Transplant Scenario}

The shortage of human organs for transplantation is a serious problem, and is exacerbated by the fact that current organ selection processes discard a significant number of organs deemed non-viable (not suitable) for transplantation. The organ viability assessment illustrates the ubiquity of disagreement and conflict of opinion in the medical domain. What may be a sufficient reason for discarding an organ for some qualified professionals may not be for others. Different policies in different hospitals and regions exist, and a consensus among medical professionals is not always feasible. Hence, contradictory conclusions may be derived from the same set of facts. For example, consider a donor with a smoking history but no chronic obstructive pulmonary disease (COPD). The medical guidelines indicate that a donor's smoking history is a sufficient reason for deeming a donor's lung as non-viable. However, there are qualified physicians that reason that the donor's lung is viable given that there is no history of COPD [9]. Similarly, the guidelines suggest discarding the kidney of a donor whose cause of death was

\footnotetext{
${ }^{1}$ Arguments deemed weaker than a given threshold are not accepted.
} 
streptococcus viridans endocarditis (sve). However, by administrating penicillin to the recipient this means that the kidney can safely be transplanted.

Currently, the decision to offer for transplantation or discard an organ available for transplantation, is based solely on the assessment of doctors at the donor site (Donor Agent, $D A$ ). This organ selection process does not account for the fact that: 1) medical doctors may disagree as to whether an organ is viable or non-viable; 2) different policies in different hospitals and regions exist, and; 3) viability is not an intrinsic property of the donor's organ, but rather, an integral concept that involves the donor and recipient characteristics as well as the courses of action to be undertaken in the transplantation process [9]. In particular, current organ selection process allow for a $D A$ to discard an organ that medical doctors at the recipient site (Recipient Agents, $R A$ ) may claim to be viable and, given the chance, could provide strong arguments to support this claim.

In [13] a novel organ selection process is proposed in which ProCLAIM is used to coordinate joint deliberation between donor and recipient agents in order to prevent the discard of organs due to the application of inappropriate organ acceptability criteria. This helps reduce the disparity between the demand for and supply of organs.

ProCLAIM is thus instantiated with the proponent agents being the $D A$ and $R A$, the Guideline Knowledge encodes the donor and organ acceptability criteria consented by the transplant organizations, i.e. the criteria the medical doctors should refer to when deciding the organs' viability 2 The Argument Source Manager relates to the agents' reputation. Namely, the $M A$ may deem as stronger the arguments submitted by agents with good reputation (e.g. a $R A$ that has in the past successfully transplanted those organs which he claimed to be viable). Finally, the CBRe allows the $M A$ to evaluate the submitted arguments on the basis of past recorded transplantation cases (see [15]).

In [14] a first attempt to formalise the ASR was presented, through a protocol-based exchange of arguments. However, argument schemes in that formalisation have to be constructed in a somewhat ad-hoc fashion, hindering the application of ProCLAIM in new scenarios (e.g. [6]). In the following section we introduce a simple dialogue game with six moves, corresponding to six argument schemes respectively. We intend to use these abstract scheme as a basis for the construction of the ASR (see future work).

\section{Arguing over Action Proposals}

In this section we introduce six abstract schemes that additionally represent six basic dialogue moves (later, in $\$ 5$, we discuss how they can be extended). Our starting point is Atkinson's et. al. argument scheme over action [4]:

In the current circumstances $\mathbf{R}$ we should perform action $\mathbf{A}$ to achieve new circumstances $\mathbf{S}$ which will realise some goal $\mathbf{G}$ which will promote some value ${ }^{3}$

\footnotetext{
${ }^{2}$ Transplant organizations periodically publish the consented organ acceptability criteria. However, these criteria rapidly evolve because of the researchers' effort in extending them to reduce organ discards. Hence, the more advanced transplant units deviate from consented criteria.

${ }^{3}$ In this sense values represent the social interests promoted through achieving the goal. Thus they are qualitative, as opposed to quantitative, measures of the desirability of a goal.
} 
Currently in ProCLAIM the appropriateness of an action is only evaluated with respect to one value as the agents are all reasoning so as to promote one value shared by all (i.e. the recipients' quality of life). Thus, the model does not take into account other possible values, such as the financial impact of the proposed action. Therefore, the value element of this argument scheme can be ignored for the purposes of this paper.

Another assumption of the model is that proposed actions are presumed to be appropriate, given some minimum context. Hence, to deem an action inappropriate agents must successfully argue that the proposed action has some undesirable side effect. That is, that the action realises some undesirable goal (in the transplant scenario, for example, these account for: severe_infection, graft_failure, cancer, etc).

Hence, agents in favor of the proposed action argue that NO undesirable goal will be realised. While, agents against the proposed action argue that an undesirable goal will be realised $4 \mathrm{We}$ formalise these proposals as follows:

Definition 1. An argument is represented in this dialogue game as a tuple:

$$
<\text { Context, Fact, Prop_Action, Effect, Neg_Goal > }
$$

Where Context is a set of facts that are not under dispute, that is, assumed to be true. Fact is a set of facts such that given the context Context, then the proposed action (or set of actions) Prop_Action result in a set of states Effect that realises some undesirable goal Neg_Goal. Fact and Ef fect may be empty sets and Neg_Goal may be equal to $\mathrm{nil}$, representing that no undesirable goal is realised. So, arguments in favor of a proposed action are of the form: < Context, Fact, Prop_Action, Ef fect, nil > whereas arguments against a proposed action, for instance against a transplantation of an organ, highlight some negative goal that will be realised: e.g. $<$ Context, Fact, Prop_Action, Effect,severe_infection $>$

Hence, the arguments used in the dialogue take into account: 1) the current state of affairs referenced by the facts deemed relevant by the proponent agents; 2) the proposed actions; 3 ) the new state achieved if a proposed action is undertaken, and; 3) the undesirable goals which the new state realises.

In ProCLAIM, a proposed action (e.g. transplant an organ) is deemed appropriate if there are no expected undesirable effects. Thus, a proposed action is by default assumed appropriate. Nonetheless, there must be some minimum set of conditions for proposing such an action (e.g. an available organ and a potential recipient for that organ). Thus, the dialogue starts by submitting an argument that claims the appropriateness of an action and the subsequent dialogue moves will attack or defend the presumptions present in that argument by claiming there is (resp. there is not) an undesirable side effect.

The six schemes we now introduce are partial instantiation of the more general scheme introduced in definition 1. These more specific schemes are intended to help identify the legal instantiation of the more general scheme at each stage of the dialogue.

\footnotetext{
${ }^{4}$ Other accounts making use of undesirable outcomes (goals to be avoided) exist in the literature, e.g. Bench-Capon and Prakken's account [7, which is focused on the concept of accrual, and Amgoud and Kaci's account [1], which is defined for use in negotiation dialogues. The aims of these two accounts differ from ours, thus we will make no further use of them in this paper.
} 
Let us consider $R$ and $S$ to be finite sets of facts in the current state and the resultant state respectively. Let $A$ be a finite set of actions and $G^{-}$a finite set of undesirable, or negative, goals (nil $\in G^{-}$). Let the elements of $R, S$ and $A$ be represented as predicates with grounded variables (e.g. donor (john, lung) $\in R$ ). Let, also, elements in $G^{-}$be represented as propositions (e.g. severe_infection).

Thus, a dialogue starts with the submission of the argument:

AS1: $<m_{-} c,\{\}, p_{-} a,\{\}, \operatorname{nil}>$

Where $m_{-} c \subseteq R$ is a minimum set of facts that an agent requires for proposing a nonempty set of actions $p_{\_} a \subseteq A$. For example, if a lung of a donor $d$ (donor ( $\left.d, l u n g\right)$ ) is available for a potential recipient $r$ (pot_recip ( $r$, lung ) ), the argument for transplantation (transp ( $r$, lung ) ) instantiates AS1 as follows:

$A=<\{$ donor (d, lung), pot_recip (r, lung) $\},\{\},\{\operatorname{transp}(r, 1$ ung $)\}$, \{\}$, \mathrm{nil}>$

Associated with the argument scheme are CQs that question the justification presented in an instantiated argument. In particular, to $A S 1$ is associated the CQ 5 :

AS1_CQ1: Is there a contraindication for undertaking the proposed action?

The CQs identify the following dialogue moves by instantiating argument schemes that correspond to the posing of the CQs. Thus, associated with AS1_CQ1 is the scheme:

AS2: $<m_{-} c, r 1, p_{-} a, s 1, g^{-}>$, where $r 1 \subset R, s 1 \subset S$ and $g 1^{-} \in G^{-} /\{\mathrm{ni} 1\} \quad(r 1$ and $s 1$ nonempty). That is, $\mathbf{A S 2}$ identifies contraindications $r 1$ for undertaking $p_{\_} a$.

For relevance and consistency we require that the facts in $r 1$ are non redundant nor in conflict with those in $m_{-} c$. That is, that $r 1$ is consistently relevant w.r.t to $m_{-} c$.

Definition 2. Let $B$ be a nonempty set, then we say that $B$ is consistently relevant w.r.t the set $A$, iff: given a background theory $\Gamma$ with a consequence relation $\vdash$ then:

1) $\forall b \in B, A \nvdash_{\Gamma} b$ and 2) $A \cup B \nvdash_{\Gamma} \perp$

In the simplest case $(\Gamma=\emptyset)$, this amounts to $b \notin A$ and $\neg b \notin A, \forall b \in B$.

Let us suppose that the donor of the offered lung has smoking history $\left(\mathrm{d}_{-} \mathrm{p}\left(\mathrm{d}, \mathrm{s} \_\mathrm{h}\right)\right)$. Let us suppose as well that the $D A$ believes $s_{\_} h$ is a contraindication because it may lead to a graft failure. The agent can submit an $\operatorname{argument} B 1$ attacking $A$ by instantiating AS2 as follows (see fig. 3, Example 1): $r 1=\left\{d_{-} p\left(d, s \_h\right)\right\} ; s 1=\{$ reject $(r$, lung) $\} ; \quad \mathrm{g} 1^{-}=\{$graft_failure .

Associated with AS2 are three CQs that in turn identify three argument schemes:

AS2_CQ1: Are the current circumstances such that the stated effect will be achieved?

\footnotetext{
${ }^{5}$ The CQs associated with the scheme enable agents to attack the validity of the various elements of the scheme and the connections between them. Also, there may be alternative possible actions and side effects of the proposed action. Only a subset of the CQs for arguing over action proposals are relevant in this particular application. The full list can be found in [4].
} 


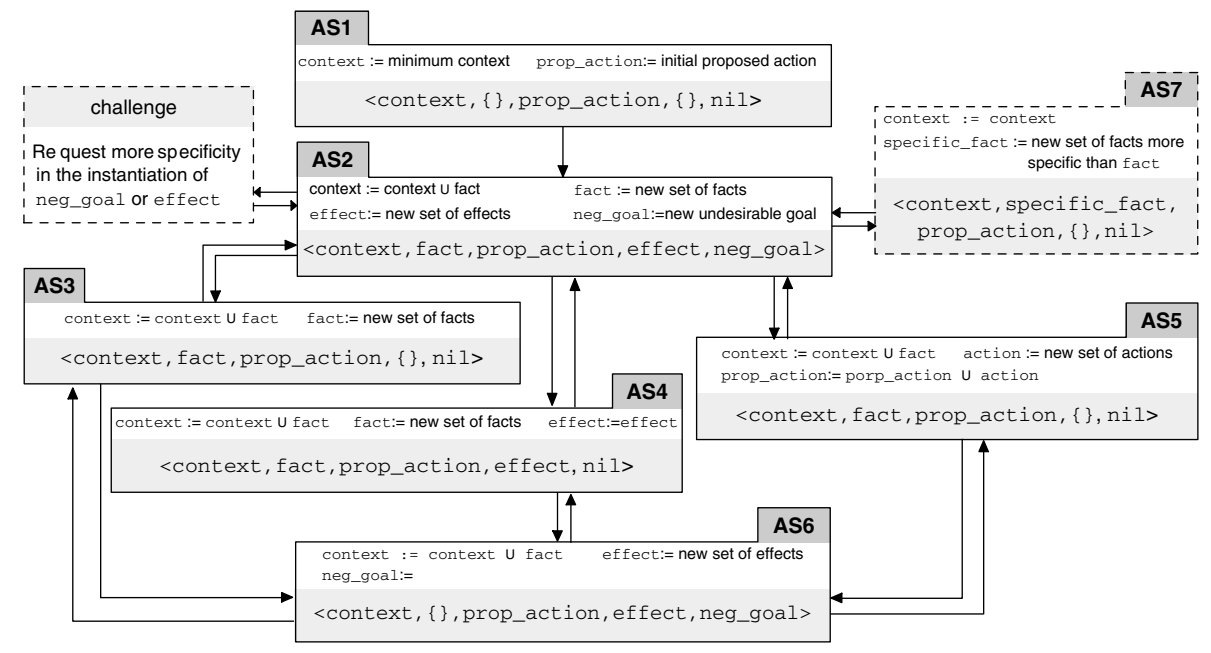

Fig. 2. Schematic representation of the dialogue game

AS3: $<m_{-} \cup \bigcup r 1, r 2, p_{-} a,\{\}, \mathrm{nil}>$, where $r 2 \subset R$ is consistently relevant w.r.t. the updated context $m_{-} c \bigcup r 1$.

AS2_CQ2: Are the current circumstances such that the achieved effect will realise the stated goal?

AS4: $\quad<m_{-} \cup \bigcup r 1, r 2, p_{-} a, s 1, \mathrm{nil}>$, where $r 2 \subset R$ is consistently relevant w.r.t. the updated context $m_{-} c \bigcup r 1$.

AS2_CQ3: Is there a course of action that prevents the achievement of the stated effect?

AS5: < $m_{\_} c \bigcup r 1,\{\}, p_{\_} a \bigcup a, s 1$, nil $>$, where $a \subset A$.

Supposing the $R A$ believes smoking history is not a contraindication because the donor did not have chronic obstructive pulmonary disease $(\operatorname{codp})$, by instantiating scheme AS3 with $r 2=\left\{\neg \mathrm{d} \_\mathrm{p}\right.$ ( d, copd) $\} R A$ can submit $\operatorname{argument} C 1$ (see fig. 3) that attacks $B 1$ (see fig.4 4 ). Figure 2 depicts a schematic representation of the dialogue game.

Arguments instantiating schemes AS3, AS4 or AS5, are of the form < context, fact, prop_act, effect, nil >, with fact and effect possibly empty. These arguments introduce either a new fact or a new "prophylactic" action that may in turn warrant, respectively cause, some undesirable secondary effect. Hence, associated with these schemes is the CQ:

AS3,4,5_CQ1: Will the introduced factor cause some undesirable side effects?

AS6: < context $\bigcup$ fact, \{\} , prop_action, $s 2, g 2^{-}>$, with $s 2 \subseteq S$, nonempty, and $\exists \alpha \in s 2$ such that $\alpha \notin s 1$ (see fig. 3 Example 2, for an illustration).

An argument instantiating AS6 can in turn be attacked by arguments instantiating AS3, AS4 or AS5. Also, an argument instantiating AS3, AS4 or AS5, alter either the 


\begin{tabular}{|c|c|c|}
\hline ID & Type & Argument \\
\hline A & AS1 & $<\{$ donor(d,lung), pot_recip(r,lung) $\},\{\},\{\}$, transp(r,lung), \{\} , nil $>$ \\
\hline B1 & AS2 & $<\{$ donor(d,lung), pot_recip(r,lung $)\},\left\{d \_p\left(d, s \_h\right)\right\}$, prop_act, $\{$ reject(r,lung $\left.)\right\}$, graft_failure $>$ \\
\hline $\mathrm{C} 1$ & AS3 & $<\{$ donor(d,lung), pot_recip(r,lung $)\} \cup\left\{d \_p\left(d, s \_h\right)\right\},\left\{d \_p\left(d, n o \_c o p d\right)\right\}$, prop_act, \{\} , nil $>$ \\
\hline B2 & AS2 & $<\{$ donor(d,lung), pot_recip(r,lung)\}, \{d_p(d,hiv)\}, prop_act, $\{$ recip_p(r,hiv)\}, sever_infect $>$ \\
\hline $\mathrm{C} 2$ & AS4 & $<$ context U \{d_p(d,hiv)\}, \{p_r_p(r,hcv)\}, prop_act, \{rec_p(r,hiv)\}, nil $>$ \\
\hline D2 & AS6 & 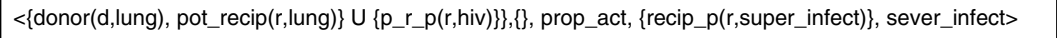 \\
\hline B3 & AS2 & $<\{$ donor(d,lung), pot_recip(r,lung)\}, \{d_p(d,sve)\}, prop_act, \{recip_p(r,svi)\}, sever_infect $>$ \\
\hline C3 & AS5 & $<\{$ donor(d,lung), pot_recip $(r$, lung $)\} \cup\left\{d \_p(d, v e)\right\},\{\}$, prop_act $U\{\operatorname{treat}(r$, penicillin $)\},\{\}$, nil $>$ \\
\hline D3 & AS2 & $<\{$ donor(d,lung), pot_recip(r,lung $)\},\left\{p \_r \_p\left(r, p e n \_a l l e r g y\right)\right\}$, prop_act, $\{$ recip_p $(r$,anaphylaxis $)\}$, sever_infect $>$ \\
\hline
\end{tabular}

Fig. 3. Three example dialogues resulting from the submission of an argument $A$ proposing transplantion of a lung (transp ( $r$, lung $)$ ) to a potential recipient $r$ (pot_recip $(r$, lung $)$ ). Figure 4 . depicts the argument interaction. Example 1: Argument $B 1$ attacks $A$ by identifying smoking history, a property of the donor $\left(\mathrm{d}_{-} \mathrm{p}\left(\mathrm{d}, \mathrm{s} \_\mathrm{h}\right)\right)$, as a contraindication, causing an eventual rejection in the recipient $(r e j e c t(r, l u n g))$ that realises the negative goal of graft failure. However, argument $C 1$ attacks $B 1$ claiming that this consequence will not be achieved because the donor does not have a chronic obstructive pulmonary disease ( $\neg$ d_p $(d, c o p d)$ ). Example 2: Argument $B 2$ identifies HIV as a contraindication ( $\mathrm{d}_{-} \mathrm{p}(\mathrm{d}, \mathrm{hiv})$ ) that causes the recipient to also develop HIV, a severe infection. $C 2$ claims that the potential recipient already has HIV (p_r_p $(r, h i v))$ and so for that recipient, HIV will not be a severe infection. Argument D2, attacks $C 2$ by stating that if the donor and potential recipient have HIV, a consequence of the transplant is a superinfection. Finally, in example 3, argument $B 3$ identifies the contraindication streptococcus viridans endocarditis in the donor $\left(\mathrm{d} \_\mathrm{p}(\mathrm{d}, \mathrm{sve})\right)$ which will result in a streptococcus viridans infection in the recipient ( $\left.r_{-} \mathrm{p}(r, \mathrm{svi})\right)$. Argument $C 3$ states that this infection can be prevented if the recipient is treated with penicillin (treat ( $r$, penicillin)). However, argument $D 3$ attacks the appropriateness of the action by stating that the potential recipient is allergic to this antibiotic (p_r_p ( $r$, pen_allergy)), thus causing anaphylaxis in the recipient ( $r \_p$ ( $r$, anaphylaxis)).

context or proposed action set. Hence, as depicted in figure 2 this argument can be attacked by an argument instantiating AS2.

A submitted argument in the dialogue attacks the argument it replies to. However, some arguments, in particular, those that claim the proposed action realises some undesirable goal (i.e. arguments instantiating AS2 and AS6) will counter-attack an attack made on the argument (i.e., the arguments symmetrically attack). Hence, the nature of the attack between arguments in the dialogue differs depending on the argument schemes they instantiate (see fig. 4 a). Broadly speaking, the rationale as to whether argument $A$ symmetrically or asymmetrically attacks $B$, is that in the latter case $B$ is an argument in favor of a proposed action that makes an assumption that there is no contraindication. $A$ contradicts this assumption. In the former symmetric case, there is a disagreement (it is a moot point) as to whether the newly introduced factor is or is not a contraindication for the proposed action (see [11] for more details on the motivation). 

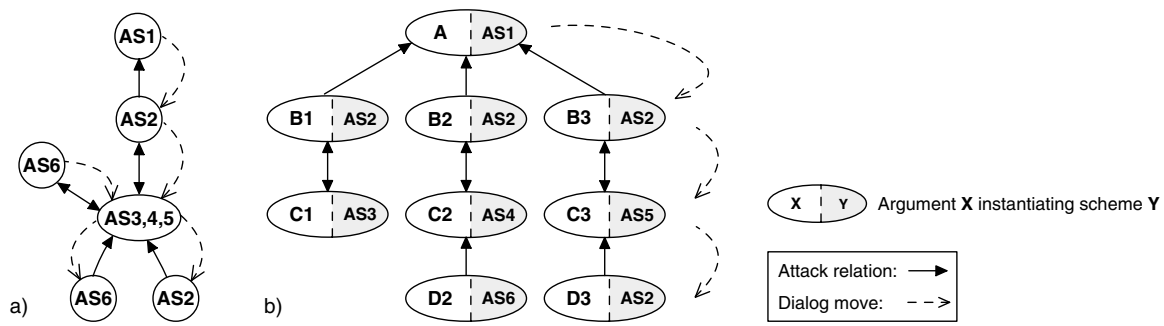

Fig. 4. a) Attack relation between the Argument Schemes. b) Dialogue graph illustrating the interaction between the arguments used in the three examples given in figure 3

\section{Future Work}

In domains with uncertainty agents may not always be able to provide a precise account of the expected effects of an action and thus, the negative goal these may realise. Thus agents may require terms with different levels of specificity. However, if the specificity relation is introduced in $R, S, A$ and $G^{-}$, new argument-based dialogue moves become relevant. For example, in figure 2 two additional dialogue moves were proposed: 1 ) A challenge move that requires agents to be more specific on the terms used. 2) If a fact (e.g. cancer on the donor) is identified as causing an undesirable effect (cancer on the recipient) via scheme AS2, in order to attack this argument the scheme AS7 can be instantiated with a more specific account of the introduced fact (e.g. non systemic cancer) which does not cause the stated undesirable effect (although cancer is in general a contraindication, the donor has a cancer which is not systemic, where the latter, more specific, is not a contraindication).

We also intend to extend the dialogue game so as to allow for arguing over beliefs. In particular, to question whether an introduced fact is indeed the case (e.g. does the donor actually have a smoking history?).

Currently we are working on a methodology for constructing the ProCLAIM's Argument Scheme Repository. The idea is to further specialise the introduced argument schemes to construct a repository of domain dependent schemes.

\section{Conclusions}

In this paper we have proposed a dialogue game for deliberating over action proposals. This dialogue game is intended to (although not restricted to) 1) formalise the protocolbased exchange of arguments in ProCLAIM ; 2) provide a basis for the construction of the Argument Scheme Repository. The dialogue can be regarded as a specialisation of that proposed by Atkinson et al. in [4]. The specialisation is required in order provide an implementation that ensures that all the relevant factors can be elicited from the proponent agents [14] in order to decide whether it is safe to undertake the proposed action, taking into account that the dialogue participants may disagree.

The ProCLAIM model provides for practical realisation of the dialogue game by referencing knowledge sources that describe the schemes and critical questions, ensure that instantiations of the schemes are valid, and bring to bear the results of previous 
dialogues (cases) and agent reputations in order that the relative strengths of arguments exchanged during the dialogue can be evaluated.

Acknowledgments. This research was supported in part by EC Project ASPIC (FP6IST-002307).

\section{References}

1. Amgoud, L., Kaci, S.: On the generation of bipolar goals in argumentation-based negotiation. In: Rahwan, I., Moraïtis, P., Reed, C. (eds.) ArgMAS 2004. LNCS (LNAI), vol. 3366, pp. 192-207. Springer, Heidelberg (2005)

2. ASPIC: Deliverable d2.1: Theoretical frameworks for argumentation (June 2004), http: / / www . argumentation.org/Public_Deliverables.htm

3. Atkinson, K.: What Should We Do?:Computational Representation of Persuasive Argument in Practical Reasoning. PhD thesis, Department of Computer Science, University of Liverpool, UK (2005)

4. Atkinson, K., Bench-Capon, T., McBurney, P.: Computational representation of practical argument. Synthese 152(2), 157-206

5. Atkinson, K., Bench-Capon, T., McBurney, P.: A dialogue game protocol for multi-agent argument over proposals for action. Autonomous Agents and Multi-Agent Systems 11, 153$171(2005)$

6. Aulinas, M., Tolchinsky, P., Turon, C., Poch, M., Cortés, U.: Is my spill environmentally safe? towards an integrated management of wastewater in a river basin using agents that can argue. In: WATERMATEX (May 2007)

7. Bench-Capon, T., Prakken, H.: Choosing what to do by accruing arguments. In: Conference on Computational Models of Natural Argument. Frontiers in Artificial Intelligence and Applications, vol. 144, pp. 247-258. IOS Press, Amsterdam (2006)

8. Dung, P.M.: On the acceptability of arguments and its fundamental role in nonmonotonic reasoning, logic programming and $n$-person games. Artificial Intelligence 77, 321-357 (1995)

9. López-Navidad, A., Caballero, F.: Extended criteria for organ acceptance: Strategies for achieving organ safety and for increasing organ pool. Clin Transplant, Blackwell Munksgaard 17, 308-324 (2003)

10. McBurney, P., Parsons, S.: Dialogue game protocols. In: Communication in Multiagent Systems, pp. 269-283 (2003)

11. Modgil, S., Tolchinsky, P., Cortés, U.: Towards formalising agent argumentation over the viability of human organs for transplantation. In: Mexican International Conference on Artificial Intelligence 2005, pp. 928-938 (2005)

12. Nealon, J., Moreno, A.: The application of agent technology to healthcare. In: 1st International Joint Conference on Autonomous Agents and Multiagent Systems (2002)

13. Tolchinsky, P., Cortés, U., Modgil, S., Caballero, F., López-Navidad, A.: Increasing humanorgan transplant availability: Argumentation-based agent deliberation. IEEE Intelligent Systems 21(6), 30-37 (2006)

14. Tolchinsky, P., Modgil, S., Cortés, U.: Argument schemes and critical questions for heterogeneous agents to argue over the viability of a human organ. In: AAAI 2006 Spring Symposium Series; Argumentation for Consumers of Healthcare, pp. 105-111. AAAI Press, Stanford, California, USA (2006)

15. Tolchinsky, P., Modgil, S., Cortés, U., Sànchez-Marrè, M.: CBR and Argument Schemes for Collaborative Decision Making. In: Conference on Computational Models of Natural Argument, vol. 144, pp. 247-258. IOS Press, Amsterdam (2006)

16. Walton, D.N.: Argument Schemes for Presumptive Reasoning. Lawrence Erlbaum Associates, Mahwah, NJ, USA (1996) 